\title{
Acyl-CoA Dehydrogenase, Short-Chain Deficiency
}

National Cancer Institute

\section{Source}

National Cancer Institute. Acyl-CoA Dehydrogenase, Short-Chain Deficiency. NCI

Thesaurus. Code C84539.

A genetic disorder characterized by deficiency of the enzyme short-chain acyl-coenzyme A dehydrogenase that metabolizes short-chain fatty acids. Signs and symptoms appear in infancy or childhood and may be triggered during fasting or illness. They include vomiting, hypoglycemia, lethargy, hypotonia and failure to thrive. 